\title{
Prejuízos fisiológicos causados pela perda dentária e relação dos aspectos nutricionais na Odontogeriatria
}

\author{
Physiological damage caused by tooth loss and relationship of nutritional aspects in Pediatric \\ Dentistry \\ Daño fisiológico por pérdida dentaria y relación de aspectos nutricionales en Odontopediatría
}

\section{Resumo}

A população de idosos no Brasil tem crescido em ritmo acelerado e o cirurgião dentista deve estar atento a ampliar seus conhecimentos na área da odontogeriatria para proporcionar um tratamento correto, eficaz e com conforto ao paciente idoso, já que estes apresentam alterações fisiológicas, condições sistêmicas, psicológicas e sociais decorrentes do processo de envelhecimento. O objetivo desta revisão de literatura é discutir as alterações na qualidade de vida do paciente idoso, incluindo seu reflexo emocional e efeitos psicológicos. Para tanto, foi utilizado uma pesquisa qualitativa de cunho bibliográfico de fonte secundária, método indutivo para análise e elaboração do presente trabalho. Uma das alterações é a perda dentária que tem efeitos significativos na saúde dos idosos, pois afeta a capacidade mastigatória, restringem o consumo de vários alimentos, dificultam a fonação, além dos danos estéticos. A má absorção dos nutrientes gera um desequilíbrio nutricional com prejuízo para realização e manutenção de processos orgânicos vitais, maior susceptibilidade a enfermidades e dificuldade na recuperação de doenças. A alimentação adequada pode levar a uma velhice saudável com maior capacidade funcional e menor incidência de doenças, melhorando a qualidade e estilo de vida.

Palavras-chave: Odontogeriatria; Alterações fisiológicas; Qualidade de vida.

\begin{abstract}
The elderly population in Brazil has grown at a fast pace and the dentist must be attentive to expand his knowledge in the field of odontogeriatrics to provide a correct, effective and comfortable treatment for the elderly patient, since they present physiological changes, systemic conditions, psychological and social consequences of the aging process. The purpose of this literature review is to discuss changes in the quality of life of elderly patients, including their emotional reflex and psychological effects. For that, a qualitative research of bibliographic nature from a secondary source was used, an inductive method for the analysis and elaboration of the present work. One of the changes is tooth loss, which has significant effects on the health of the elderly, as it affects masticatory capacity, restricts the consumption of various foods, makes phonation difficult, in addition to aesthetic damage. Malabsorption of nutrients generates a nutritional imbalance with impairment for the realization and maintenance of vital organic processes, greater susceptibility to
\end{abstract}


illnesses and difficulty in recovering from diseases. Adequate nutrition can lead to a healthy old age with greater functional capacity and less incidence of disease, improving quality and lifestyle.

Keywords: Odontogeriatrics; Physiological changes; Quality of life.

\section{Resumen}

La población anciana en Brasil ha crecido a un ritmo acelerado y el odontólogo debe estar atento para ampliar sus conocimientos en el campo de la odontogeriatría para brindar un tratamiento correcto, efectivo y cómodo para el paciente anciano, ya que presentan cambios fisiológicos, condiciones sistémicas, Consecuencias psicológicas y sociales del proceso de envejecimiento. El propósito de esta revisión de la literatura es discutir los cambios en la calidad de vida de los pacientes ancianos, incluidos sus reflejos emocionales y sus efectos psicológicos. Para ello se utilizó una investigación cualitativa de carácter bibliográfico a partir de una fuente secundaria, método inductivo para el análisis y elaboración del presente trabajo. Uno de los cambios es la pérdida de dientes, que tiene efectos importantes en la salud de las personas mayores, ya que afecta la capacidad masticatoria, restringe el consumo de diversos alimentos, dificulta la fonación, además de daño estético. La malabsorción de nutrientes genera un desequilibrio nutricional con impedimento para la realización y mantenimiento de procesos orgánicos vitales, mayor susceptibilidad a enfermedades y dificultad para recuperarse de enfermedades. Una nutrición adecuada puede conducir a una vejez saludable con mayor capacidad funcional y menor incidencia de enfermedades, mejorando la calidad y el estilo de vida.

Palabras clave: Odontogeriatría; Cambios fisiológicos; Calidad de vida.

\section{Introdução}

Werner e colaboradores (1998) define a odontologia geriátrica como o ramo da odontologia que enfatiza o cuidado bucal da população idosa, especificamente tratando do atendimento preventivo e curativo de pacientes com doenças ou condições de caráter sistêmico e crônico associados a problemas fisiológicos, físicos ou patológicos.

No processo de envelhecimento o corpo humano sofre alterações fisiológicas consideráveis, sendo necessário que o Cirurgião-Dentista tenha ciência dessas mudanças uma vez que a maioria delas acomete a saúde bucal. É necessário conhecer as alterações fisiológicas e patológicas que acometem o organismo do paciente idoso, bem como os aspectos psicossociais para este indivíduo. O profissional deve manter contato com o médico Geriatra, para avaliar administração de drogas, visando o idoso que faz uso de medicação podem apresentar efeitos colaterais e provocar alterações em sua saúde geral (Rosa et al., 2010).

O edentulismo ou perda dentária pode ser considerado como produto final da morte dental, consequência da cárie, doença periodontal não controlada, e estes são considerados pela sociedade como algo natural do envelhecimento (Haikal et al., 2011).

A atividade mastigatória desempenha um papel muito importante na hemostasia do corpo inteiro. Problemas odontológicos podem influenciar a qualidade de vida e do bem-estar do paciente. Os dentes são essenciais para o processo de mastigação e eles desempenham um papel crítico na nutrição geral e na saúde geral, pois diminuindo a capacidade mastigatória, irá dificultar e limitar o consumo de diversos alimentos. Além disso, a perda dentária pode afetar a fonação, estética e originar alterações psicológicas, contribuindo para reduzir a qualidade de vida do paciente idoso (Borges et al., 2011).

No Brasil, o último levantamento epidemiológico mostra que os brasileiros na faixa etária de 65 a 74 anos já perderam 93\% dos seus dentes (Ministério da Saúde, 2012). Isso revela a precariedade da saúde bucal na Odontogeriatria e denuncia a falta de cuidados que tiveram ao longo da sua vida.

Sendo assim, o Cirurgião-Dentista deve se preocupar com o bem-estar do seu paciente como um todo, por meio de uma visão ampla, pois essa população traz consigo um conjunto de patologias sistêmicas que interferem na saúde bucal, instruir-se sobre os cuidados específicos que essa faixa etária necessita.

O objetivo desse trabalho foi identificar e analisar o edentulismo na terceira idade nas quais são trazidos contigo várias alterações para sua saúde em geral, como forma de conscientizar a população em geral para cuidados com sua saúde bucal e danos trazidos pela perda dentária. 


\section{Metodologia}

Trabalho baseado em uma revisão de literatura por meio de artigos científicos pesquisados com estudos que abordam os prejuízos fisiológicos causados pela perda dentária na Odontogeriatria. Foram pesquisados artigos nas bases bibliográficas Scielo, Biblioteca Virtual de Saúde (BVS), Pubmed e Google acadêmico, e empregados os termos "perda dentária, prejuízos fisiológicos, nutrição e Odontogeriatria", com artigos em inglês e português. Foram selecionados para o levantamento bibliográfico os artigos mais ressaltantes ao termo de escolha, entre um período de 1998 a 2019.

\section{Revisão de Literatura}

O Brasil passa por um processo de envelhecimento populacional rápido e intenso, tanto que a expectativa de vida do brasileiro continuará aumentando nas próximas décadas. Por isso, há necessidade de se proporcionar maior qualidade de vida ao segmento idoso da população, enfocando os aspectos físico, social e psicológico (Unluer, 2007).

É necessário conhecer as alterações fisiológicas e patológicas que acometem o organismo do paciente idoso. Algumas enfermidades comuns ao paciente idoso apresentam consequências bucais para as quais o cirurgião-dentista deve estar atento, a fim de minimizar no tratamento odontológico. O avançar da idade promove um aumento na prevalência de algumas doenças bucais, visto que a mucosa oral torna-se mais susceptível aos danos mecânicos (Vaccarezza et al., 2017).

A saúde bucal, parte integrante e inseparável da saúde geral dos indivíduos, algumas vezes são tratadas com descaso, devido o fato de grande parcela desse grupo ser edêntula (Rosendo et al., 2017). A atenção médica e odontológica para esta população deve estar centralizada essencialmente na melhoria da qualidade de vida, com especial atenção à prevenção de doenças e outras disfunções. A fim de se obter maior efetividade nas ações de saúde bucal para os idosos, é importante avaliar a auto percepção que estes pacientes têm em relação aos impactos das condições de saúde bucal na sua qualidade de vida (Barbosa, 2011).

A negligência nos procedimentos de higiene oral bem como as visitas não periódicas aos consultórios odontológicos, e as alterações fisiológicas, como a perda de elasticidade da mucosa, sofridas pelos idosos são fatores que podem provocar o desenvolvimento de afecções bucais (Vasconcelos et al., 2012). A maioria dos problemas odontológicos observados na população da terceira idade está relacionada com complicações patológicas adquiridas no decorrer da vida destes pacientes, oriundas de uma ineficiente higiene bucal, iatrogenia de profissionais, falta de orientação, e de acesso aos serviços de saúde, seja por negligência pessoal ou condição socioeconômica (Lelis et al., 2009). Dentre as alterações percebidas na cavidade oral do paciente idoso algumas decorrentes de manifestações de doenças sistêmicas, deficiência nutricional, efeito colateral de determinados medicamentos, que repercutem no funcionamento das glândulas salivares, na saúde do tecido periodontal, e da mucosa oral. Destas o edentulismo, a cárie dentária, doença periodontal, a xerostomia, redução da capacidade gustativa, e as lesões nos tecidos moles são as que apresentam maior prevalência e acometimento na saúde bucal do idoso (Catão et al., 2011). A seguir relacionam-se algumas condições clínicas fisiológicas que podem estar presentes na cavidade bucal do idoso.

O Edentulismo é caracterizado por perda dos elementos dentários, acarretando ao paciente uma série de problemas no quesito funcional, psicológico e social, refletindo de maneira direta na alimentação do paciente bem como em sua qualidade de vida. Muito dos problemas que acometem a população idosa vem de complicações de processos patológicos acumulados no decorrer da vida do indivíduo (Lelis et al., 2009). O edentulismo ainda é aceito pela sociedade como algo normal e natural com o avançar da idade, e não como reflexo da falta de programas preventivos e cuidados com sua saúde bucal. A ausência dentária leva o paciente a manter uma dieta mais pastosa, rica em carboidratos fato este que proporciona um aumento de peso corporal, e consequentemente pode levar ao desenvolvimento de doenças sistêmicas como a obesidade, a hipertensão arterial, diabetes entre outras. A perda da dentição permanente influenciará na mastigação e, consequentemente, na digestão, bem como na gustação, na pronúncia e na estética (Andrade Acevedo et al., 2011). 
Pode-se considerar que um indivíduo com todos os dentes tem uma capacidade de mastigação de $100 \%$. Com a perda de um dente, essa capacidade passa a ser de $70 \%$, podendo chegar a $25 \%$ com o uso de próteses totais. A perda de parte da capacidade mastigatória em pacientes portadores de prótese e da sensibilidade mastigatória não está apenas relacionada com o uso de aparelhos protéticos, mas também às condições periodontais satisfatórias desses pacientes (Borges et al., 2011). Levantamento epidemiológico realizado mostra que os brasileiros na faixa etária de 65 a 74 anos já perderam $93 \%$ dos seus dentes (Ministério da Saúde, 2012).

Dados mundiais revelam que a cárie é a doença bucal mais comum entre os indivíduos com sessenta anos ou mais. Fatos como a redução no fluxo salivar decorrente o uso de determinados medicamentos, dificuldade motora no processo de higienização e as alterações na dieta potencializam de forma direta os efeitos da doença (Fernandes-Costa et al., 2013).

Assim como o processo carioso, a doença periodontal, é decorrente ao acúmulo da placa bacteriana na superfície dos dentes, acarretando em destruição dos tecidos adjacentes. Mudanças ocorridas na dieta e uma diminuição na quantidade de saliva promovem um acúmulo mais rápido de biofilme na superfície dentária de pacientes idosos. Com o avanço da idade também promove uma alteração gradual no tecido ósseo, com diminuição do tecido mineralizado, onde a reabsorção aumenta e a neoformação óssea diminui, resultando em um aumento da porosidade óssea. Como consequência a doença periodontal promove a perda dentária, diminuindo a capacidade mastigatória do paciente, repercutindo diretamente nas funções do sistema estomatognático, além do desenvolvimento de halitose, que interfere na relação social deste com os indivíduos que convive (Vasconcelos et al., 2012).

O paciente idoso sofre uma perda de $30 \%$ da capacidade funcional das glândulas salivares, havendo consequentemente uma diminuição no processo de lubrificação da cavidade oral, tornando o indivíduo mais susceptível ao desenvolvimento de condições patológicas (Silva et al., 2008). Thomson et al., (2011) concluiu que a prevalência e incidência de xerostomia nos idosos estavam fortemente associadas ao uso de medicamentos e também a dificuldades na mastigação, deglutição, gustação e fala, o que resulta em dieta pobre e má nutrição, diminuindo a interação social.

A redução da capacidade gustativa associada ao doce, amargo e ácido é verificada a partir dos cinquenta anos e atinge $80 \%$ dos pacientes idosos. A gustação sofre alterações com o avanço da idade porque o número de botões gustativos diminui significativamente, principalmente após os setenta anos (Rosendo et al., 2017).

Alterações metabólicas que ocorrem no processo de envelhecimento podem gerar a perda da morfologia gengival, tornando a mucosa oral atrofiada e friável. Essas alterações resultam na diminuição da capacidade de recuperação dos tecidos bucais e redução do suprimento sanguíneo prejudicando o processo de regeneração tecidual. A estomatite protética causada por desadaptação protética é uma afecção de alta prevalência e apresentada com grande frequência por pacientes idosos. O câncer é uma doença que afeta, em sua maioria, pacientes de sexo masculino, entre 50 e 60 anos, e que apresentam o hábito tabagista e etilista (Roldan et al., 2003).

Traumas psicológicos do edentulismo evidenciam o impacto da reabilitação oral na percepção da autoimagem e, consequentemente, seus resultados na qualidade de vida de uma pessoa idosa (Medeiros et al., 2014). A autoestima está relacionada com a imagem corporal, principalmente por padrões estéticos impostos pela sociedade. É visto que as perdas dentárias causam alterações físicas na face. Por exemplo, pode-se observar depressão da comissura labial e da base do nariz, perda do tônus muscular, redução da altura vertical do terço inferior da face e aprofundamento das linhas de expressão, além do aparecimento de reações psicológicas, que incluem perda de autoconfiança, preocupação com a aparência e autoimagem. Todos esses problemas fazem com que o indivíduo mantenha em segredo a perda do dente (Okoje et al., 2012).

A reabilitação do edêntulo deve visar não somente a reposição dos dentes perdidos, mas também proporcionar além de conforto e função mastigatória, a estética almejada por ele. Esta pode ser o fator-chave para seu sucesso físico e emocional, a reabilitação com próteses totais para estabelecer função e estética contribuindo para a integração social (Papadaki, 2012). 
O envelhecimento está relacionado com alterações fisiológicas que afetam a necessidade de vários nutrientes. Dentre as principais alterações fisiológicas que podem afetar o comportamento alimentício dos pacientes idosos, podemos evidenciar a diminuição da sensibilidade para os gostos primários, a perda parcial ou total dos elementos dentários, a desaceleração do metabolismo e a presença de doenças crônicas com consequente utilização de múltiplos medicamentos. Sabe-se que a maioria dos idosos apresenta alterações sensoriais, com declínio da visão, da audição, do olfato e da gustação. Principalmente os dois últimos afetam diretamente a nutrição desses indivíduos, uma vez que o alimento passa a ser menos apetitoso e desejado pelo idoso. Alterações gastrointestinais podem ocorrer principalmente devido à alteração na estrutura e na função do estômago. Como consequência há diminuição da secreção salivar, redução da motilidade gástrica, queda na produção de suco e hormônios gástricos e enzimas digestivas, além de poder ocorrer diverticulite, constipação intestinal que são resultados de uma nutrição pobre em fibras alimentares (Bhutto et al., 2008).

A nutrição e saúde bucal são dois fatores que se complementam, um afetando diretamente o outro. Da mesma forma que a falta de dentes irá levar a uma mastigação comprometida e muitas vezes com declínio da qualidade nutricional, esse declínio nutricional irá levar a problemas bucais e à perda de mais dentes, formando assim um círculo vicioso e altamente prejudicial à saúde geral do idoso. Diante disso, é importante observar e identificar possíveis carências alimentares que possam trazer problemas bucais, evitando-se a perda de dentes, assim como orientar os pacientes parcial ou totalmente edêntulos sobre a importância de se adaptarem a uma dieta adequada, que seja possível de acordo com sua condição bucal e capacidade mastigatória. A função mastigatória pode ser restaurada por terapia protética adequada, que resulta em aumento na atividade dos músculos mastigatórios durante a mastigação, reduzindo-se o tempo e o número de movimentos até a deglutição (Carvalho et al., 2020; Coelho et al., 2009).

A avaliação do estado nutricional do idoso é considerado complexa em razão da influência de uma série de fatores que necessitam ser investigados detalhadamente. Alterações fisiológicas, processos patológicos crônicos e situações individuais que ocorrem com o envelhecimento, geralmente interferem no estado nutricional do indivíduo. Dentre os problemas bucais mais comuns que podem ser agravados pela má alimentação, estão as cáries, a doença periodontal, a perda de dentes e a perda da integridade da mucosa sendo essa, prejudicada em muitos casos de avitaminoses (Coelho et al., 2009; Vaccarezza et al., 2017).

Ausência da vitamina A, tem como consequência descamação da mucosa, prejuízo no reparo dos tecidos, aumento do risco de candidíase, hipertrofia gengival e inflamação, leucoplasia, diminuição do paladar, xerostomia. Suas fontes estão no fígado de aves e animais, cenoura. Ausência da vitamina C: (leve): maior risco de infecção, aumento dos sinais e sintomas periodontais, cicatrização lenta. (severa): escorbuto. Fontes: laranja, limão, abacaxi. Ausência da vitamina K: aumento no risco de sangramento e candidíase. Fontes: fígado e verduras. Ausência da vitamina D: regeneração óssea anormal, calcificação incompleta dos dentes e do osso alveolar. Suas fontes são encontradas no óleo de peixe e gema de ovos. (Coelho et al., 2009).

$\mathrm{O}$ fato dos idosos ficarem sem próteses ou com próteses inadequadas acarreta problemas estéticos, funcionais, nutricionais, gastrointestinais e remodelação da ATM, com dores orofaciais e dificuldade de alimentação por diminuição da habilidade mastigatória, o que leva a uma dieta inadequada, pois preferem alimentos macios e pobres em nutrientes, com efeito grave sobre seu estado nutricional. A alimentação desempenha papel importante na prevenção de doenças. Esses pacientes podem desenvolver doenças sistêmicas como câncer no intestino, diabetes, hipertensão e doenças cardiovasculares pelo consumo exagerado de gorduras saturadas, dieta pobre em fibras, antioxidantes e caroteno (Mattar, 2014).

A preservação e a recuperação da função mastigatória representam um dos principais objetivos de um tratamento odontológico reabilitador, já que a capacidade mastigatória alterada impossibilita ou modifica as atividades de alimentação. A maioria dos edêntulos encara a perda dental como consequências inevitáveis do envelhecimento e não como um problema que mereça ou possa ser corrigido (Haikal et al., 2011). O uso da prótese dentária aumenta a capacidade de mastigação, peso corporal e qualidade de vida relacionada à saúde bucal e estética. A prótese permite a esperança de recuperação de uma aparência 
desejável. Um belo sorriso pode mostrar simpatia, alegria e autoconfiança. Tem uma crescente busca de pacientes edêntulos, não apenas por uma reabilitação que devolva função, mas também que lhes devolva um belo sorriso, e a substituição do estado edêntulo para o dentado deve ser acompanhada de técnicas e critérios que visam à satisfação desse paciente (Abreu et al., 2012).

Antes do desenvolvimento de implantes dentários, a única opção era próteses totais, porém estudos mostram que a reabilitação oral com implantes dentários proporciona melhor retenção e estabilidade às próteses. Para uma população com menos recursos muitas vezes a única alternativa são as próteses convencionais para a sua reabilitação, porém é necessário melhorar esse tratamento sendo que grande parte dos pacientes reabilitados com essas próteses são incapazes de adaptar a elas, sendo indicado a odontologia implantar para que esses pacientes possam ter uma melhor função mastigatória (Borges et al., 2011).

O rosto é a parte mais diferenciada do corpo e está indissociavelmente ligado ao indivíduo e a sua identidade. Os dentes são considerados aspectos importantes nas relações empregatícias, sociais além de influenciar na autoestima das pessoas. A beleza física é algo almejado dentro da sociedade, e pra que esse desejo seja cumprido, o dentista deve estar ciente da preocupação de que o paciente tem em relação a sua aparência, e se esforçar para atender as exigências estéticas, proporcionando àquele paciente função e estética e consequentemente na melhora da saúde geral, a sua qualidade de vida (Hantash et al., 2011).

\section{Discussão}

Autores como Vaccareza et al. (2017), Barbosa (2011), Vasconcelos (2012), Hantashi et al., (2011), afirmaram que o paciente idoso apresenta consequências bucais a quais o cirurgião-dentista deve estar atento, como conhecer que as alterações fisiológicas e patológicas a fim de minimizar desenvolvimento de afecções bucais, e proporcionar maior qualidade de vida a esses pacientes.

A perda dentária causa vários problemas para sua saúde em geral e afeta a alimentação diretamente, pois o paciente vai ingerir alimentos pastosos, consumo maior de carboidrato trazendo consequências para sua saúde geral (Lelis et al., 2009; Andrade Acevedo et al., 2011).

Os pacientes além de sessenta anos possuem a cárie dental cada vez mais frequente devido à diminuição do fluxo salivar, na qual esse paciente sofre uma perda de $30 \%$ da capacidade funcional das glândulas salivares, e está associada ao uso de medicamentos (Thomson et al., 2008; Fernandes-Costa et al. 2013, Silva et al., 2008).

A partir dos cinquenta anos é reduzida a capacidade de gustação, assim como alterações metabólicas prejudicando também a regeneração tecidual. (Roldan et al.2003, Rosendo et al., 2017).

Autores como Papadaki (2012), Okoje et al. (2012), Mattar (2014), visam a reabilitação do idoso não só como a reposição dos dentes mas que proporcione estética, função e conforto à eles, proporcionando a eles autoestima, qualidade de vida visando a prevenção de doenças.

Bhutto e Morley (2008), Coelho et al., (2009), Bhutto et al. (2008), afirmam que a saúde bucal e nutrição estão intimamente ligados, um afetando o outro. A ausência dentária leva a uma mastigação comprometida, afetando a nutrição destes indivíduos trazendo consigo alterações gastrointestinais, diminuindo a qualidade nutricional, e com isso pode gerar a perda de mais dentes.

A ausência de vitaminas no nosso organismo pode trazer grandes alterações na cavidade oral do paciente idoso, como ausência da vitamina $\mathrm{A}$, causando prejuízo na reparação dos tecidos; ausência da vitamina $\mathrm{C}$, tendo maior risco à infecção, cicatrização retardada; ausência da vitamina $\mathrm{D}$, calcificação dos dentes incompleta e ausência da vitamina $\mathrm{K}$ trazendo consequências como aumento do risco de sangramento e candidíase (Coelho et al., 2009).

Um dos principais objetivos da reabilitação oral são a preservação e recuperação da função mastigatória já que esta alterada modifica as atividades de alimentação. Diante disso, temos a reabilitação com o uso da prótese dentária permitindo uma 
aparência desejável, possibilitando uma condição bucal satisfatória (Haikal et al., 2011; Abreu et al., 2012).

Borges et al., (2011) afirma que antes a única opção que tínhamos para a reabilitação oral seria com próteses convencionais, porém a odontologia implantar está cada vez mais presente na atualidade e trazendo consigo muitos benefícios em relação a prótese dentária. A odontologia implantar proporciona melhor retenção e estabilidade às próteses e consequentemente melhor função mastigatória.

\section{Considerações Finais}

A qualidade do envelhecimento é resultado do estilo de vida pelo qual o indivíduo optou, sendo os hábitos alimentares responsáveis por grande parcela da qualidade dessa etapa vivida.

A alimentação adequada pode levar a uma velhice saudável com maior capacidade funcional e menor incidência de doenças. A má absorção dos nutrientes gera um desequilíbrio nutricional com prejuízo para realização e manutenção de processos orgânicos vitais, maior susceptibilidade a enfermidades e dificuldade na recuperação de doenças.

A literatura reporta a necessidade de realização de mais estudos, buscando soluções adequadas que permitam o estabelecimento de políticas públicas de atenção a saúde oral, possibilitando uma melhor qualidade de vida a população idosa.

\section{Agradecimentos}

CAPES - 001 .

\section{Referências}

Abreu, C. W. de, \& Munhoz, E. (2012). Os fatores que influenciam na satisfação do paciente submetido a tratamento de prótese total convencional. $H U$ Revista, 37(4). https://periodicos.ufjf.br/index.php/hurevista/article/view/1559

Andrade Acevedo, R., Henrique Carvalho Batista, L., Sandini Trentin, M., \& Awad Shibli, J. (2011). Tratamento periodontal no paciente idoso. Revista Da Faculdade De Odontologia - UPF, 6(2). https://doi.org/10.5335/rfo.v6i2.1635

Barbosa K. G. N. (2011) Condições de saúde bucal em idosos: uma revisão da realidade brasileira. Odontol. Clín.-Cient. (Online). 12(42), 681-694, http://revodonto.bvsalud.org/scielo.php?script=sci_arttext\&pid=S1677-38882011000300007

Bhutto, A., \& Morley, J. E. (2008). The clinical significance of gastrointestinal changes with aging. Current opinion in clinical nutrition and metabolic care, 11(5), 651-660. https://doi.org/10.1097/MCO.0b013e32830b5d37

Borges, T., Mendes, F. A., de Oliveira, T. R., Gomes, V. L., do Prado, C. J., \& das Neves, F. D. (2011). Mandibular overdentures with immediate loading: satisfaction and quality of life. The International journal of prosthodontics, 24(6), 534-539.

Carvalho, G. A. O., Ribeiro, A. de O. P., Câmara, J. V. F., \& Pierote, J. J. A. (2020). Abordagem odontológica e alterações bucais em idosos: uma revisão de literatura. Research, Society and Development, 9(7), e938975142. https://doi.org/10.33448/rsd-v9i7.5142

Catão, M. H. C. V., Xavier A. F. C., (2011) O impacto das alterações do sistema estomatognático na nutrição do idoso. Revista de Atenção à Saúde - RAS. 9(29) https://doi.org/10.13037/rbcs.vo19n29.1349

Coelho et al. (2009) Nutrição e Saúde Bucal. In: Campostrini E. Odontogeriatria. Revinter, Cap 7, 38-67.

Fernandes-Costa, A. N., Vasconcelos, M. G., Queiroz, L. M. G., Barboza, C. A. G., \& Vasconcelos, R. G. (2013). As principais modificações orais que ocorrem durante o envelhecimento. Revista Brasileira De Ciências Da Saúde, 17(3), 293-300. https://periodicos.ufpb.br/ojs/index.php/rbcs/article/view/13650

Haikal, D. S. et al. (2011) Self-perception of oral health and impacto n quality of life among the elderly: a quantitative-qualitative approach. Ciênc. saúde coletiva, 16(7), 3317-3329, http://dx.doi.org/10.1590/S1413-81232011000800031.

Hantash, R. O., AL-Omiri, M. K., Yunis, M. A., Dar-Odeh, N., \& Lynch, E. (2011). Relationship between impacts of complete denture treatment on daily living, satisfaction and personality profiles. The journal of contemporary dental practice, 12(3), 200-207. https://doi.org/10.5005/jp-journals-10024-1035

Lelis E.R., Rocha E. S., Mattar D. D. S., Martins C. R., Silva D. D., Souaa de Sousa M. L. R., et al. (2009) Incidência e prevalência de doenças bucais em pacientes idosos: Alterações morfológicas, sistêmicas e bucais. . Rev Inpeo Odontol 3(2), 82-47.

Mattar, D.D.S. (2014) Capacitação profissional de odontogeriatrias não acompanham crescimento da demanda de pacientes idosos. Rev Gutierre odontolife; 60. 22 .

Medeiros, Safira Lince de, Pontes, Marília Pinheiro de Brito, \& Magalhães Jr., Hipólito Virgílio. (2014). Autopercepção da capacidade mastigatória em indivíduos idosos. Revista Brasileira de Geriatria e Gerontologia, 17(4), 807-817. https://doi.org/10.1590/1809-9823.2014.13150 
Research, Society and Development, v. 10, n. 1, e45810111730, 2021

(CC BY 4.0) | ISSN 2525-3409 | DOI: http://dx.doi.org/10.33448/rsd-v10i1.11730

Ministério da Saúde. (2012). Secretaria de Atenção à Saúde -Departamento de Atenção Básica. In Caderno de atenção domiciliar (Vol. 1). Brasília, DF

Okoje, V. N., Dosumu, O. O., Alonge, T. O., \& Onyeaso, C. (2012). Tooth loss: are the patients prepared? Nigerian journal of clinical practice, 15(2), $172-175$. https://doi.org/10.4103/1119-3077.97305

Papadaki, E., \& Anastassiadou, V. (2012). Elderly complete denture wearers: a social approach to tooth loss. Gerodontology, 29 2, e721-7.

Roldán, S., Herrera, D., \& Sanz, M. (2003). Biofilms and the tongue: therapeutical approaches for the control of halitosis. Clinical oral investigations, 7(4), 189-197. https://doi.org/10.1007/s00784-003-0214-7

Rosa, L., Zuccolotto, M. C., Bataglion, C., \& Coronatto, E. (2010). Odontogeriatria - a saúde bucal na terceira idade. Revista Da Faculdade De Odontologia $U P F$, 13(2). https://doi.org/10.5335/rfo.v13i2.599

Rosendo, R. A. et al. (2017) Autopercepção de saúde bucal e seu impacto na qualidade de vida em idosos: uma revisão de literatura. RSC. https://doi.org/10.1590/S1415-52732004000400010

Silva, S., Rossie, H., Lima, P., Lamey, O., Veloso, M., Souza, M., et al. (2008). Saúde bucal do idoso institucionalizado em dois asilos de Passo Fundo - RS. RGO 56(3):303-30.

Thomson, W.M., et al. (2011). Shortening the Xerostomia Inventory. Oral Surgery, Oral Medicine, Oral Pathology, Oral Radiology, and Endodontology, 112(3), $322-327$.

Unlüer, S., Gökalp, S., \& Doğan, B. G. (2007). Oral health status of the elderly in a residential home in Turkey. Gerodontology, 24(1), 22-29. https://doi.org/10.1111/j.1741-2358.2007.00136.x

Vaccarezza, G., Fuga, R., \& Ferreira, S. (2017). Saúde bucal e qualidade de vida dos idosos. Revista de Odontologia da Universidade Cidade de São Paulo, 22(2), 134 - 137. https://doi.org/10.26843/ro_unicid.v22i2.407

Vasconcelos, L. C. A., Prado Júnior, R. R., Teles, J. B. M., \& Mendes, R. F. (2012). Autopercepção da saúde bucal de idosos de um município de médio porte do Nordeste brasileiro. Cadernos de Saúde Pública, 28(6), 1101-1110. https://doi.org/10.1590/S0102-311X2012000600009

Werner, C. W., Saunders, M. J., Paunovich, E., et al. (1998) Odontologia Geriátrica. RFO, 13(2), 82-86. 